\title{
Vivienda contemporánea e instalaciones: entre la objetividad tecnológica y la subjetividad del proyecto arquitectónico
}

\section{Contemporary Housing facilities: between the technological objectivity and the subjectivity of the architectural Project}

Rodolfo Bellot, Diego Fiscarelli

\begin{abstract}
Docente Investigador UNL. Coordinador técnico del LATMAT (Laboratorio de Técnicas y Materiales). Profesor titular ordinario de Instalaciones I, II y III. Facultad de Arquitectura, Diseño

y Urbanismo.
\end{abstract}

Docente Investigador categoría V - UNLP. Becario Posdoctoral Consejo Nacional de Investigaciones Científicas y Técnicas CONICET. Laboratorio de Tecnología y Gestión Habitacional LATEC Facultad de Arquitectura y Urbanismo.

rjbellot@fadu.unl.edu.ar, dfiscarelli@fau.unlp.edu.ar

\section{RESUMEN}

El presente artículo aborda la relación entre las instalaciones y la vivienda, tema trabajado por diversos autores. En primera instancia se definen los conceptos y sus orígenes para luego explicar los diferentes momentos de esa relación caracterizados por continuidad o ruptura.

Esta relación se describe a partir de diferentes periodos, con un punto de inicio en consonancia con la segunda Revolución Industrial continuando con una historiografía crítica que intenta reconocer los diferentes momentos en los cuales el desarrollo tecnológico se incorporó al proyecto de la vivienda en donde hasta nuestros días ha tenido afinidades y desencuentros en una discusión que aún no ha sido saldada.

\section{ABSTRACT}

This article approaches the relationship between facilities and housing, a topic studied by various authors. In the first instance, the concepts and their origins are defined to later explain the different moments of that relationship characterized by continuity or rupture.

This relationship is described from different periods, with a starting point in line with the second Industrial Revolution, continuing with a critical historiography that tries to recognize the different moments in which technological development was incorporated into the housing project, where even today days he has had affinities and disagreements in a discussion that has not yet been settled.

PALABRAS CLAVE: Instalaciones; Vivienda; Tecnología; Sustentabilidad.

KEYWORDS: Facilities; Housing, Technology; Sustentability

FECHA DE RECEPCIÓN: 14/9/2021 | FECHA DE ACEPTACIÓN: 8/11/2021

DOI: http://dx.doi.org/10.30972/arq.0185671 


\section{INTRODUCCIÓN}

El vínculo de la arquitectura para con la tecnología ha intensificado su debate en los últimos años, específicamente revisando las acciones e innovaciones que se produjeron durante el siglo XX. Numerosas investigaciones se enfocan en estudiar la relación entre diseño y técnica, dando cuenta de la incidencia de la tecnología sobre la disciplina (Carpo y Lemerie, 2005; Picon, 2008), incluso algunas desde revisiones históricas (Chang y Winter, 2015; Medina Warmburg et al. 2015; Requena-Ruiz, 2016), con una mirada que supone una postura superadora de la mera explicación de la maquina tal cual lo entendía el Movimiento Moderno. Se parte del supuesto, que el proyecto de las instalaciones, no debe considerar a éstas como meras tuberías por las que circulan fluidos, sino como un sistema, parte de otro mayor, que permite el acondicionamiento y la transferencia de energías, fusionándose en el diseño de toda la obra. En tal sentido se entiende por instalaciones a todo el conjunto que complementa -o suplementa- a los sistemas pasivos, abarcando entonces, desde los vitales servicios de recursos, los indispensables servicios mecánicos de suministros, los elementos tecnológicos y todos aquellos que permiten al hombre habitar un espacio que no sería posible solamente con los elementos físicos de la estructura, el cerramiento o la adecuación natural. "Sin instalaciones, la mayor parte de los edificios contemporáneos no cumplen la utilitas vitruviana del siglo XXl" (Martín-Gómez, 2006 p. 4)

Según lo entiende Díaz Dorado (2008), las instalaciones sanitarias, contra incendio, higrotérmicas, de ventilación, iluminación y acústicas son inherentes al bienestar y la salud, siendo una función de la arquitectura el crear espacios aptos para el hábitat, por lo que otorga al conocimiento de las instalaciones una condición prioritaria. En un texto canónico, Instalaciones aplicadas a los edificios el Arquitecto Julio Cesar Lemme (1973), expresa el mismo concepto al sostener que las instalaciones edilicias se entienden como una necesidad básica para preservar la salud de las personas y conservar su higiene.

En el ámbito específico de la práctica proyectual, el Arquitecto Rem Koolhaas (1992) expresa que las instalaciones son pertinentes al proyecto arquitectónico, al sostener que ellas comprenden un porcentaje significativo del costo de un edificio (más del $30 \%$ ) y por ende deben ser comprendidas desde la génesis del diseño. En esta misma línea de pensamiento, autores como Tombesi (2017) y Martin-Gómez (2015) sostienen que las instalaciones se deben concebir con integración al proyecto, no como un complemento, sino como parte de él, integrando los espacios con los sistemas tecnológicos.

Es posible verificar que diversos expertos, en las últimas décadas, han abordado esta línea de trabajo, considerando a las instalaciones como intrínsecas al proyecto, siendo sistemas que deben proyectarse desde la complejidad, desde el acondicionamiento, la eficiencia y el ambiente sostenible. (Czajkowski y Gómez, 2011; Alonso-Frank, Kuchen y Alamino-Naranjo, 2015; San Juan, 2013 y Fillipin, 2018) reflexionando sobre el rol de las instalaciones en relación a los recursos, parten de que éstas, junto a otros elementos del proyecto, se vinculan directamente con el control de las energías, adecuando los edificios con el medio y considerando además el prioritario rol de los profesionales y usuarios, que con sus decisiones y hábitos influyen sobre éstos. Al mismo tiempo, se concuerda con del arquitecto de-Garrido (2014) quien expresa que el proyecto de las instalaciones, entendidas como sistemas activos, deben pensarse junto al adecuado diseño arquitectónico de las envolventes y materiales (sistemas pasivos). 
Bellot, Fiscarelli

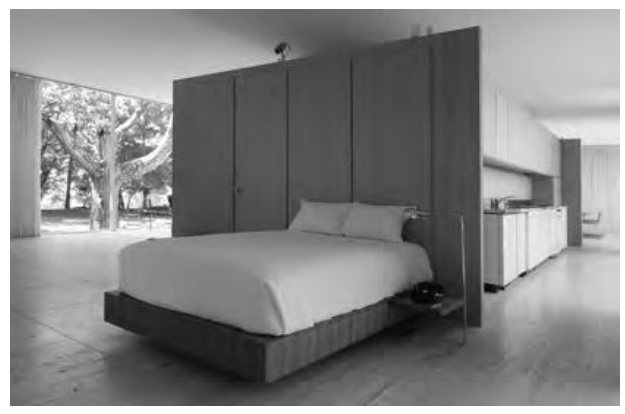

Figura 1. Espacialidad entorno al núcleo de servicios de la casa Farnsworth. Fuente: Valencia y Carvajal (2013).

\section{Historia de una antigua relación}

En nuestro país, los orígenes del saneamiento se ubican aproximadamente en el año 1822, en donde se intenta sin éxito dotar a la ciudad de Buenos Aires de agua corriente. Fue finalmente en el año 1887, y luego de la epidemia de cólera, que se estableció el punto de partida en las obras de saneamiento.

Podemos ver entonces, como la sociedad moderna, símbolo del progreso indefinido, las libertades personales e ilustración, se nutrió de grandes descubrimientos científicos, industrialización y tecnología, los cuales produjeron nuevos entornos físicos y claro está, arrasando otros, aun en un proceso abierto, no definitivo, según lo expresa Latour (2017). Al respecto, y de acuerdo al criterio del filósofo Marshall Berman, ser modernos es vivir en un mundo de contradicciones y paradojas, es un dualismo permanente. En este escenario la tecnología se transforma en un vasto conjunto de conocimiento y saberes que le son propios a la sociedad y con los cuales va creando y modificando la realidad.

La práctica proyectual en el diseño de la vivienda en implicancia con las instalaciones, redefine en la arquitectura moderna su mayor potencial, si observamos, por ejemplo, la arquitectura de Mies Van Dr Rohe, en la casa Farnsworth (1946-1950), su obra manifiesto (fig. 1), se puede ver como se proyectó la complejidad técnica de instalaciones y servicios en un solo componente, y a partir de la compacidad de ése núcleo, cobro lugar la planta libre. Similar situación es posible observar en el conjunto de viviendas de Weissenhof (1925-1927). En palabras del propio Mies (citado en Neumeyer 1996 p.396) "Si nos limitamos a configurar solo el baño y la cocina como espacios constantes, debido a las instalaciones, y optamos por dividir el resto de la superficie habitable con paredes móviles, creo que se puede satisfacer cualquier requisito de habitabilidad". Este enlace entre instalaciones y el proyecto de la vivienda, encuentra en las últimas décadas un nuevo giro: del paradigma moderno mecanicista, a un posicionamiento energéticamente sustentable, asociado a la economía de recursos y la protección del medio.

\section{Entre la positividad tecnológica y subjetividad del proyecto}

La historia de la arquitectura fue la de un campo disciplinar que se debatió entre la técnica y el arte, el diseño y la tecnología, en nuestro interés: entre las instalaciones y el proyecto del hábitat Si bien es prácticamente imposible describir las numerosas aristas sobre el tema, en relación a obras, autores, corrientes y críticos, se intentará mediante algunos aspectos relevantes construir una historiografía que permita vislumbrar los hechos y a la vez, actuar como lentes con los cuales conducir el texto. Se trata entonces de como la positividad tecnológica puede -o ha podidoimbricarse con la subjetividad del acto creativo, de reflexionar sobre el sentido de la tecnificación en un período que inicio en el siglo XIX, pero en el XX cobró un protagonismo exacerbante y aún hoy, continua en discusión. 
En relación al tema, Vitta (2021) expresa que no hubo quizás en toda la cultura occidental un lugar en donde los extremos, el de estética y el de la técnica, se hayan enfrentado como a comienzo del siglo pasado y en la cual "[...] ambos buscaron la supremacía y del que ambos salieron profundamente modificados [...]" (p. 28). El surgimiento de la industrialización, producto de la revolución industrial y de la sociedad industrial, produjo un arte propio, que gracias a la técnica intentaba despegarse o despojarse de los cánones tradicionales.

El filósofo Alexandre Koyré cuando intentaba definir el origen de la ciencia moderna, en relación a si ésta era nueva o una continuidad de la edad media, expresaba que era una quaestio disputata ${ }^{1}$, ya que encontraba tanto defensores de una evolución continua como detractores que plantean una revolución, siendo ambas cosas verdaderas en algún punto.

Con las instalaciones y la arquitectura pasa algo similar, es cierto que existe una continuidad evolutiva y también es cierto que existen revoluciones, entre ellas la planteada luego de la revolución industrial, tal vez la que más destaca.

\section{a) Etapa previa a la plena mecanización}

Se elige el termino etapa previa a la era de la máquina, haciendo alusión al clásico texto: La mecanización toma el mando (1948) ${ }^{2}$, del historiador de arquitectura Siegfried Giedion, el cual nombró como el periodo de plena mecanización al que se desarrolla entreguerras, esto es desde 1918 a 1939.

El proceso de industrialización, fue agudizando -negativamente- las condiciones de vida de los sectores o clases obreras que en su mayoría se establecían en las urbes, situación que como bien explica (Benévolo, 2010), da origen a la urbanística moderna, y lo hace desde los defectos de la ciudad industrial y gracias a los higienistas ${ }^{3}$. Independientemente de las condiciones insalubres y de hacinamiento de las clases trabajadoras, ningún sector social escapa de los riesgos que presentan las enfermedades como el cólera y la tuberculosis, entre otras enfermedades infeccionas derivadas de la insalubridad o simplemente del desconocimiento de la ciencia para esa época.

En ese contexto, hacia fines del siglo XIX e inicios del XX, en las viviendas el cuarto de baño era un lujo, solo posible para las clases privilegiadas, en tanto que los sistemas sanitarios estaban en desarrollo e intentando superar algunas insatisfacciones.

De esta manera, los cambios en este campo fueron paulatinos, con una evolución lenta, pero constante. En primer lugar, se iniciaron en las clases sociales acomodadas y luego en las más populares, como bien es sabido, la producción de bienes industrializados de alcance masivo comenzó bien avanzado el siglo XX, en donde el mobiliario higiénico y funcional -en parte obra de los arquitectos de la Bauhaus y sus seguidores- fue expandiéndose hasta penetrar en los edificios y la vivienda (Capel, 2002). En palabras de Giedion: "Alrededor de 1920, la mecanización abarcó la esfera doméstica; por vez primera toma posesión de la vivienda y de todo lo que en ella fuese susceptible de mecanización, es decir, la cocina y el baño y sus respectivos equipos [...]" (1978 p. 56)

\footnotetext{
${ }^{1}$ El filósofo francés -ruso de nacimiento-A. Koyré en el texto Estudios de historia del pensamiento científico (1977), la considerada por este trabajo es la $24^{\circ}$ edición del año 1997, desarrolla una particular interpretación de los orígenes de la ciencia moderna, encontrando en A. C. Crombie la mejor posición de continuidad.

${ }^{2}$ La edición en castellano considerada para este trabajo es la de (1978). La mecanización toma el mando. Barcelona: Gustavo Gili

3 El higienismo, en el sentido de una corriente de pensamiento que propulsaba una amplia consideración de salud pública que incluía tanto las condiciones de las viviendas, como de los lugares de trabajo y los espacios públicos. Supuso un alto aporte e involucramiento por parte del Estado, mediante normativas, políticas sanitarias e intervenciones en el medio.
} 
Retomando los vínculos entre las instalaciones y la vivienda, es propicio considerar el concepto higienista desde una perspectiva urbana, en donde el servicio de agua y desagües, se fue consolidando hasta llegar a la denominada "ingeniería heroica" (Turton y Ohlsson,1999).

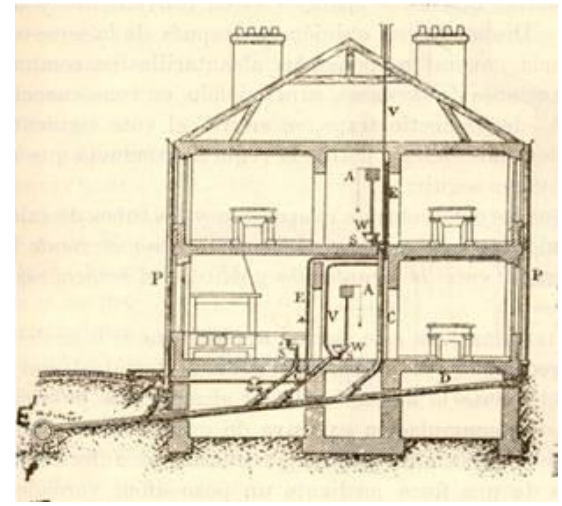

Figura 2: Eduardo Gallego Ramos (1908). Saneamiento de poblaciones. Fuente A. Buj Buj (2003).

De acuerdo a Benévolo (2010), en la relación entre las varias viviendas, es donde se pone en crisis la edificación, siendo un problema urbanístico más que constructivo.

El ingeniero militar Eduardo Gallego Ramos, luego del Primer Congreso de Saneamiento de la Habitación (París, 1904), recogió algunos datos importantes, que junto a una vasta experiencia pudo redactar en varios textos, uno de ellos denominado: Saneamiento de poblaciones (urbanas y rurales) (1908). En él, se expresa entre otros temas de interés, la discusión de cómo organizar los desagües en relación a la colectora (fig. $N^{\circ} 2$ ) -situación que implicaba profundamente el diseño interior de los proyectos edilicios de viviendas- y la importancia del baño popular con ducha, tanto de agua fría como caliente. Tal cual lo expresa (Buj Buj, 2003), un tema importante además de los desagües sanitarios era la calefacción. Eduardo Gallego Ramos (1908) los señalaba, refiriéndose al brasero, como el sistema más imperfecto higiénicamente, en cuanto a las chimeneas, (de rendimiento escaso), en tanto las estufas de carbón, (culpables de numerosas intoxicaciones), las de gas, (privativas para determinadas clases sociales), las de petróleo y alcohol, (con graves problemas en el orden higiénico), y las estufas eléctricas, la más perfecta higiénicamente pero con un empleo circunstancial, (por depender del precio del fluido), por lo general elevado para aplicarse a este uso"| (ibídem). Y en tal sentido, propondría los sistemas de calefacción colectiva mediante calderas centrales por medio de agua, aire caliente o vapor. Paralelamente a la situación expuesta, no es posible obviar que desde mediados del siglo XIX comienza junto al nuevo urbanismo y los avances de la técnica, una relación muy particular, en donde las instalaciones son protagonistas indiscutidos en los proyectos de arquitectura, se hace referencia a las construcciones en altura, que encuentran una lógica técnico|espacial que abrevará durante todo el siglo XX, encontrando en Chicago una especial connotación (Benévolo, 2010; Frampton, 2018).

\section{b) Etapa de plena mecanización}

Cono se ha mencionado, a principios del siglo XX, ya se contaba -aunque no en términos masivos- con la lámpara eléctrica, el fonógrafo, el teléfono y el ascensor. Además de estas tecnologías, aparecían mejoras y novedades en los sistemas de calefacción y saneamiento que sin lugar a duda fueron modificando la vida cotidiana en las aglomeraciones urbanas producto de la industrialización.

En el tiempo de la plena mecanización, fue cuando nacieron la mayor cantidad de necesidades en el hogar, una gran cantidad de artefactos comenzaron a utilizarse. La electricidad fue tomando popularidad, y para mediados de la segunda decena del siglo XX, ventiladores, planchas, tostadores de pan, aspiradoras y exprimidores, ya se ofrecían en catálogos, en tanto que para 
1930 la gama eléctrica y en 1932 la heladera eléctrica. (Giedion, 1978). En los edificios en altura se puede visualizar la unión entre técnica y diseño, en él desaparecieron las diferencias entre lo mecánico y lo artístico. Ábalos y Herreros (1992) afirman que para Le Corbusier y para Mies van der Rohe, los proyectos en alto representan una génesis materializada donde se podría verificar la significación arquitectónica de la técnica industrial. Para el año 1922, Le Corbusier abre un estudio con su primo Pierre Jeanneret y junto a éste en 1926 publican una serie de proclamas las cuales venían trabajando con anterioridad, que se conocen bajo el rotulo de los cinco puntos de una nueva arquitectura, a saber: los pilotis, las terrazas-jardín, la planta libre, la fenétre en longueur y la fachada libre.

El segundo punto, las terrazas jardín, presenta una característica imbricada en los términos de instalaciones y diseño, el gran maestro señala que desde larga data, los inviernos son soportados mediante una cubierta inclinada que recibe y posteriormente escurre el agua y la nieve. Este tipo de cubierta no tiene en mayor medida razón de ser. El tejado deber pasar de inclinado a plano. La idea de recoger las aguas, tanto meteóricas como del deshielo y canalizarlas por dentro, no escurriéndolas libremente mediante el techo inclinado, obedece a la utilización de hormigón armado, el cual, por su característica dilatoria ante cambios de temperatura, conserva por mayor tiempo la nieve y humedad, evitando saltos bruscos de temperatura que pudieran fisuras las cubiertas.

Esta posición conllevo, como es lógico pensar, numerosas discusiones, pero, independientemente de las limitaciones del texto escrito en 1926, es oportuno destacar el valor revolucionario que logra despojarse de toda referencia cultural y filosófica. (Benevolo, 2010)

\section{c) Segunda era de la máquina}

Desde mediados del siglo XX, la relación entre las instalaciones y el proyecto de la vivienda, guarda un lugar especial en la búsqueda de una estructura espacial que pueda albergar los sistemas de acondicionamiento mecánico, energéticos y demás sistemas de infraestructuras que se habían insinuado en los años anteriores. Reyner Banham (1975) estudio y propuso una relación entre las distintas partes componentes de la estructura, la infraestructura de servicios y aislaciones. La búsqueda de un techo falso que posibilite la existencia de redes energéticas, en donde la racionalidad y la economía coexistan son principios objetivos del espacio mecanizado. El autor, critica enfáticamente la deficiente atención que se da a las cuestiones técnicas vinculadas a los edificios en altura, "[...] temas que reciben escaso interés en la literatura histórica, que supone que todo lo que se necesitó para hacer posible los edificios elevados fueron la estructura de acero y el ascensor." (Banham 1975 p. 76). En esta posición refuerza las posibilidades otorgadas por numerosas innovaciones como el aire acondicionado, las válvulas de descarga de agua, los sistemas de presurización, el teléfono y la bujía de luz; todos elementos que brindaron soluciones a los problemas de orden ambiental generados por los espacios configurados en altura.

Banham fue quien alertó con mayor énfasis sobre la construcción historiográfica dominante, expresando que la mayoría de los textos de historia de la arquitectura, solo tratan de las formas externas, olvidando las instalaciones que posibilitan su hábitat. Por su parte, cuestiona a los maestros del Movimiento Moderno, de los cuales se horroriza y acusa por haber sacrificado el control del ambiente bajo el concepto geométrico de la estética de la era de la máquina. "Años de adoctrinarían desde que Ruskin había hecho de la tecnología un problema en vez de una oportunidad, mientras la teoría racionalista tal como era expuesta por Auguste Choisy relacionaba cambios de estilo con cambios de técnica [...]" (Banham 1975, p.131). El autor, en cambio, encuentra en la arquitectura norteamericana, las mejores expresiones de la mecanización, especialmente en la obra de Frank Lloyd Wright el mejor exponente en la conjunción del dominio formal y técnico. 
Textos tales como, Theory and design in the first machine age $(1960)^{4}$ y The architecture of the well-tempered environment (1969) ${ }^{5}$ de Banham en los cuales se analiza la relación entre los procesos técnicos y el diseño arquitectónico, venían precedidos por los ineludibles aportes de otros, como el de Sigfried Giedieon, Mechanization Takes Command (1948) y Technics and Civilization (1934) 6 de Lewis Munford. Entre ellos se puede encontrar una cartografía historiográfica que, a su manera, plantean la relación en la arquitectura desde un acercamiento -unificación- entre técnica y diseño, aunque, a decir verdad, la primera es la imperante para todos ellos.

La postura de Banham, a favor de la tecnológica, no contempla en demasía las cuestiones económicas e impactos sobre el medio. Algo similar al reclamo de Liernur (2010) por sobre la arquitectura brutalista de los Smithson, cuanto los acusa de corresponsables de la crisis ecológica global.

Ahora bien, entre aquella expresión mecanicista de Banham y lo que sobrevino después, subyace un eslabón perdido que aparece oculto bajo el concepto de sustentabilidad (Fernández Rojas, 2016). Y, en tal sentido, requiere de una redefinición del concepto de belleza, tal cual lo planeta Ábalos (2010) "sólo si hay una verdadera discusión estética, si hay una idea de belleza asociada a la sostenibilidad, ésta podrá interesar a la arquitectura de una forma no circunstancial" (p. 127)

\section{d) La crisis energética y el giro en la mecanización.}

Producto de la aparición de sistemas informáticos, el avance tecnológico y la compacidad de elementos electromecánicos, junto a la creciente demanda ambiental se comienza a experimentar un nuevo cambio, una transformación que extiende las posibilidades de las limitaciones vigentes.

Esa demanda ambiental, si bien se remonta años atrás, logra su punto de inflexión en la década del ' 70 , más precisamente con la crisis del petróleo de $1973^{7}$ que provocara una crisis económica y energética, y donde comienza a tomar fuerza el concepto de Eficiencia Energética ${ }^{8}$, esta situación conjuntamente con la evolución de una técnica creciente brindan un nuevo panorama en relación a diseño de las instalaciones en el marco del proyecto de arquitectura.

En relación a la mecanización del ambiente, la mencionada compacidad creciente de los equipos de climatización, sumado a su rápida obsolescencia, ponen en evidencia que los sistemas de pisos o plantas técnicas se encuentra en crisis, surgiendo una distribución dispersa que responde mejor a estos nuevos requerimientos. Un cambio que se busca constantemente en este período de creciente tecnificación y demandas energéticas, son las distintas soluciones constructivas entre los distintos niveles, para configurar la circulación horizontal y vertical de redes. Se hace referencia a las modificaciones del piso rígido horizontal que promovió el Movimiento Moderno y

\footnotetext{
${ }^{4}$ Edición en castellano: (1965). Teoría y diseño arquitectónico en la era de la máquina. Buenos Aires: Nueva Visión.

${ }^{5}$.Edición en castellano: (1975). La arquitectura del entorno bien climatizado. Buenos Aires: Ediciones Infinito.

${ }^{6}$ La edición en castellano considerada para este trabajo es la de (1971) Técnica y Civilización. Madrid: Alianza.

${ }^{7}$ En octubre de 1973, los países integrantes de la OPEP (países Árabes exportadores de petróleo), junto a Egipto, Túnez y Siria, decidieron no exportar más petróleo a aquellos países que habían apoyado a Israel en la guerra de Yom Kipur. Esto trajo aparejado un fuerte aumento del precio del crudo y una crisis tanto económica como energética.

8 Termino profundamente abordado en el seminario Doctoral Eficiencia Energética y Confort Térmico en el marco de la carrera por ante la FADU - UNL, dictado por el Dr. Ernesto Kutchen, del cual se desprende el concepto de NEU (nivel de eficiencia del usuario) entre otras variables que comprender el concepto de EE en edificios.
} 
que generó diversas transformaciones y diseños por un espacio estructural donde conviven lo mecánico y energético.

\section{CONCLUSIONES}

\section{Reflexiones para un futuro presente}

En tal sentido, con posterioridad a la crisis del petróleo y las preocupaciones ambientales, la relación instalaciones y vivienda, llegada a fines del siglo XX y comienzos del nuevo, se aventuran por un sendero en el cual las supra tecnologías se desarrollan sin precedentes, en tanto el diseño, encuentra nuevas expresiones. Algo que Vitta (2021) anuncia como factible siempre que se parta de la idea de que la técnica no reside en el objeto sino en su proyecto.

Al respecto, la arquitectura tiene como responsabilidad, en tanto acción que modifica el medio, constituirse en un sistema de actuación que contemple la belleza al mismo tiempo que las preocupaciones sobre el ambiente. Eficiencia energética y sostenibilidad parecieran ser conceptos implícitos de la disciplina, de la buena arquitectura. Conceptos que actualmente son superados por preceptos como el de, Carbono Neutral, un tanto confuso, especialmente si consideramos miradas antropológicas entre el mundo social y natural tal cual nos propone Latour (2017) o los apocalípticos -no por ello inverosímiles- informes de la IPCC (Panel Intergubernamental del Cambio Climático). Se sabe que carbono neural, al menos con nuestro estado de situación, es imposible de lograr, pero no por ello, deja de ser un excelente horizonte a seguir. Algo como caminar hacia el horizonte, que permite eso, avanzar, algo que no es poco. El escenario actual propone una redefinición en términos de técnica y hábitat, los avances tecnológicos presentan objetos con profundos cambios en su naturaleza, tanto en la función como en la forma. Los recursos informáticos, domótica, inmotica, electrónica y nuevas instalaciones, constituyen nuevos cuerpos cargados de complejidad. La tectónica, tal cual la entiende Frampton, algo de carácter ontológico más que representativo, está en jaque permanentemente, y en donde la arquitectura [...] "parece estar cada día más polarizada entre, por un lado un enfoque denominado high tech, basado en la producción y, por otro lado, la aportación de una fachada compensatoria para ocultar la dura realidad de este sistema universal" (2020, p. 43). Por tal motivo, nos propone sus seis puntos para una arquitectura de resistencia ${ }^{9}$ en vías de un regionalismo crítico, que asocie la relación dialéctica desde la consideración para con la naturaleza.

No se trata de una ecuación económica, tampoco de una etiqueta inmobiliaria, se trata de una ecuación que equilibre el confort, la eficiencia y la seguridad. Un justo equilibrio entre la calidad del hábitat y la calidad ambiental. La belleza de las formas y los espacios, deben incluir a la técnica, en un proceso abierto, como bucles, para significarse, para transformarlas en lo que siempre fueron, habilidades para la existencia.

\section{BIBLIOGRAFÍA}

Ábalos Vázquez I. (2010) La belleza termodinámica. En revista: 2G, revista internacional de arquitectura, N56, (p. 125) Barcelona: Gustavo Gili.

Alonso-Frank, A.; Kuchen, E. y Alamino-Naranjo, Y. (2015) Developing an energy efficiency assessment tool for buildings according to user behaviour indoors. The $31^{\circ}$ International

\footnotetext{
${ }^{9}$ Kenneth Frampton en el texto: Teoría. Los seis puntos abordados en el año 1983 y consolidados en el capítulo previo "Rappel Á L'ordre en favor de la tectónica. 1) Cultura y civilización, 2) ascenso y caída de la vanguardia, 3) el regionalismo crítico y la cultura mundial, 4) la resistencia de la forma - lugar, 5) la cultura frente a la naturaleza: topografía, contexto, clima, luz y forma tectónica, 6) lo visual frente a lo táctil.
} 
PLEA Conference Passive Low Energy Architecture: Architecture in (R)Evolution. Bologna, Italia.

Banham Reyner (1960). Theory and design in the first machine age. Nueva York: Praeger. Edición en castellano: (1965).

Banham Reyner (1969). The architecture of the well-tempered environment. Chicago: University of Chicago Press.

Benevolo. L. (2010) Historia de la arquitectura moderna. ( $8^{\circ}$ ed.). Barcelona: Gustavo Gili

Buj Buj A. (2003) La vivienda salubre: el saneamiento de poblaciones (1908) en la obra del ingeniero militar Eduardo Gallego Ramos. En Scripta Nova, revista electronica de geografía y ciencias sociales. V. VII, Nº. 146(012). Barcelona: Universitat de Barcelona.

Capel, H. (2002) La morfología de las ciudades. I. Sociedad, cultura y paisaje urbano. Barcelona: Ediciones del Serbal, 2002

Carpo, M. y Lemerie, F. (ed.). (2005). Perspective, projections, projet: techniques de la représentation architectural. París: Éditions du Patrimoine, (p.191) 〈halshs-00322813〉

Chang, J. H. y Winter, T. (2015). Thermal Modernity and Architecture. The Journal of Architecture, V 20, No 1 (p. $92-121)$.

Czajkowski, J. D., \& Gómez, A. F. (2011). Cuadernos de arquitectura sustentable. Articulos seleccionados 2011. La Plata: Universidad Nacional de la Plata.

de-Garrido, L. (2014). Arquitectura. Energía - Cero (1ํ ed.). Barcelona: Monsa.

Díaz Dorado, M. (2008). Instalaciones Sanitarias y contra incendio en edificios ( $3^{\circ}$ ed.). Buenos Aires: Alsina.

Filippín, C. (2005). Uso eficiente de la energía en edificios (1ำed.). La Pampa: Amerindia.

Frampton K. (2018) Histórica crítica de la arquitectura moderna. (4 ed.) Barcelona: Gustavo Gili

Frampton K. (2020) Kenneth Frampton: Teoría. Barcelona: Gustavo Gili

Gallego Ramos, E. (1908) Saneamiento de poblaciones (urbanas y rurales). Madrid: Imprenta del Memorial de Ingenieros

Giedion S. (1948). Mechanization takes command, a contribution to anonymous history. Nueva York: Oxford University Press.

Koollhaas R. (1992). Encontrando Libertades: conversaciones con Rem Koolhaas. Entrevista realizada por Zaera Polo A. en El Croquis № 53, p. 6 - 31.

Koyré A. (1997) Estudios de historia del pensamiento científico. (14 ed.) Mexico: Siglo XXI editores.

Latour, B. (2017). Cara a cara con el planeta. Una nueva mirada sobre el cambio climático alejada de las posiciones apocalípticas. ( $1^{\circ}$ ed.). (D. Ariel, Trad.) Buenos Aires, Argentina: Siglo Veintiuno Editores.

Lemme, J. C. (1973). Instalaciones aplicadas en los edificios ( $1^{\circ}$ ed.). Buenos Aires: Luis y Felix Fossati SRL.

Liernur J. F. (2010). En Viajes en la transición de la arquitectura española hacia la modernidad. Pozo J.M. \& García-Diego Villarías, H. (coord.) Actas del Congreso Internacional celebrado en Pamplona, Escuela Técnica Superior de Arquitectura de la Universidad de Navarra. Mayo 6 - 7. Pamplona: T6 Ediciones.

Martín-Gómez, C.. (2017). Instalaciones de acondicionamiento higrotérmico para arquitectos. Pamplona: EUNSA.

Martín-Gómez, C.. (2006). Las instalaciones y la arquitetura. En revista Tectonica №21, julio, p. 4 - 27. Madrid: ATC ediciones.

Medina Warmiburg J. (et al.) (2015). The Construction of Climate in Modern Architectural Culture, 1920-1980. Madrid: Lampreave.

Mumford L. (1934). Technics and Civilization. Nueva York: Harcourt.

Neumeyer, F. (1995). La palabra sin artificio: reflexionessobre arquitectura (1ํed.). Madrid: EI Croquis. 
Piccon, A. (2008). Notes on Modern Architecture and Technology. Revista Positions $N^{\circ} 0$ (p. 78 - 83)

Requena-Ruiz, I. (2016). Building Artificial Climates. Thermal control and comfort in Modern Architecture (1930-1960), En Ambiances: (http://ambiances.revues.org/801) (halshs01418310)

San Juan, G. A. (2013). Diseño bioclimático como aporte al proyecto arquitectonico (1ำed.). La Plata: Editorial de la Univerdiad de la Plata.

Tombesi P. (2017). En Instalaciones de acondicionamiento higrotérmico para arquitectos. MartínGomez C. Pamplona:EUNSA

Turton, A.R. \& Ohlsson, L., (1999) Water Scarcity and Social Stability: Towards A Deeper Understanding of the Key Concepts Needed To Manage Water Scarcity in Developing Countries. Paper for the 9th Stockholm Water Symposium, Sweden 9 - 12 August 1999

Valencia , J. F., \& Carvajal, E. H. (2015). El nucleo de servicios de la casa Fransworth: la planta libre en el espacio doméstico. En revista dearq № 16, p. 204 - 213.

Vitta M. (2021). El proyecto de la Belleza: el diseño entre el arte y la técnica (1 ed.). Buenos Aires: Fondo de la Cultura Económica. 